\title{
Electroweak phase transitions with BSM fermions
}

\author{
Martin Gabelmann, M. Margarete Mühlleitner and Jonas Müller \\ Institute for Theoretical Physics (ITP), Karlsruhe Institute of Technology, \\ Wolfgang-Gaede-Straße 1, D-76131 Karlsruhe, Germany \\ E-mail: martin.gabelmann@kit.edu, milada.muehlleitner@kit.edu, \\ jonas.mueller@kit.edu
}

Abstract: We study the impact of additional beyond-the-Standard Model (BSM) fermions, charged under the Standard Model $(\mathrm{SM}) \mathrm{SU}(2)_{L} \otimes \mathrm{U}(1)_{Y}$ gauge group, on the electroweak phase transition (EWPT) in a 2-Higgs-Doublet-Model (2HDM) of type II. We find that the strength of the EWPT can be enhanced by about $40 \%$ compared to the default 2HDM. Therefore, additional light fermions are a useful tool to weaken the tension between increasing mass constraints on BSM scalars and the requirement of additional light scalar degrees of freedom to accommodate a strong first order EWPT. The findings are of particular interest for a variety of (non-minimal) split supersymmetry scenarios which necessarily introduce additional light fermion degrees of freedom.

KeYWords: Supersymmetry Phenomenology

ArXIV EPRINT: 2107.09617 


\section{Contents}

1 Introduction 1

2 A 2 HDM with electroweakinos 3

3 Finite temperature corrections $\quad 4$

4 Phenomenology of the EWPT 5

4.1 The case $\xi_{c}^{2 \mathrm{HDM}}<1 \quad 6$

$\begin{array}{lll}4.2 & \text { The case } \xi_{c}^{2 \mathrm{HDM}}>1 & 9\end{array}$

$\begin{array}{lll}5 & \text { Prospects for split-SUSY } & 10\end{array}$

$\begin{array}{llr}6 & \text { Conclusions } & 11\end{array}$

\section{Introduction}

The discovery of a Higgs boson by the LHC experiments ATLAS [1] and CMS [2] was a milestone in modern particle physics and has marked a further great success of the Standard Model (SM) describing the fundamental interactions of elementary particles. However, there are still open questions that cannot be answered within the SM such as the baryon-antibaryon asymmetry. Sakharov proposed three conditions a model needs to fulfill in order to explain the dynamical production of the baryon asymmetry through electroweak baryogenesis in the early universe [3]. While the necessary baryon-violating processes are possible in the SM, the required $\mathrm{CP}$-violation and departure from thermal equilibrium are not sufficient in the SM [4-7] to explain the observed baryon asymmetry of the universe [8]. Therefore, physics beyond the SM (BSM) is needed in order to explain the baryon-anti-baryon asymmetry.

The requirement of departure from thermal equilibrium can be translated into the requirement of a strong first order electroweak phase transition (SFOEWPT). The presence of an SFOEWPT can be used as an additional theoretical constraint a benchmark scenario of a BSM model needs to fulfill. Only those parameter points that provide an SFOEWPT, are suitable candidates for electroweak baryogenesis. A minimalistic approach to enable an SFOEWPT is the extension of the scalar sector of the SM by including additional $\mathrm{SU}(2)_{L}$ doublets or singlets in the scalar potential. Despite the rich phenomenology of these models, there is an increasing difficulty to simultaneously fulfill all experimental constraints and the requirement of strong first order phase transitions, see e.g. [9].

On the other hand, there are more involved model frameworks such as supersymmetry (SUSY) that solve (some of) the problems of the SM by a symmetry relating bosonic and fermionic degrees of freedom. However, theories like the Minimal Supersymmetric SM (MSSM) have come under strong experimental pressure in the past years. In particular, LHC 
searches for colored scalars are pushing the soft-SUSY-breaking scale of top superpartners above the TeV-scale $[10,11]$. However, fermions charged under $\mathrm{SU}(2)_{L} \otimes \mathrm{U}(1)_{Y}$ are much less constrained and currently still allowed to be as light as $100-200 \mathrm{GeV}$ [12]. This lead to a paradigm shift in the construction of SUSY-breaking mechanisms and the proposal of split SUSY scenarios [13-17], which decouple all scalar superpartners of the SM fermions but maintain rather light non-SM fermions at the same time. The resulting low-energy effective field theory (EFT) often consists of a 2-Higgs-Doublet-Model (2HDM) or a 2HDM with additional light electroweak fermions and/or additional singlet fields.

The (power-suppressed) contributions from heavy superpartners to observables such as Higgs boson masses thereby can be incorporated by performing a matching of a given effective field theory (EFT) Lagrangian on a more fundamental model such as the MSSM [18-24]. This strategy is currently also applied to Dark Matter observables [17, 25-27], EWPTs [28], gravitational waves [29] and electroweak baryogenesis [30, 31] in low energy EFTs of the Next-to-MSSM (NMSSM). However, in contrast to Higgs boson mass predictions, the theoretical accuracy in the matching for the other mentioned observables is often only performed at tree-level and partially at one-loop level. Furthermore, studies of the EWPT do not always consider an extended fermion sector but rather focus on the extended scalar sector of the low-energy theory. Neglecting the additional supersymmetric fermions can in principle be justified in two different ways: (i) higgsinos/gauginos can be easily decoupled by assuming a large $\mu$-term and large soft-breaking masses; (ii) the phenomenology of the EWPT is determined by the shape of the scalar potential which is independent of the fermion sector at leading order (LO). However, it was shown in ref. [26], that the decoupling of fermions in non-minimal spit-SUSY is not necessarily radiatively stable. Therefore, argument (i) cannot always be applied. Furthermore, temperature corrections to the scalar potential are of higher order and therefore the temperature profile of a scalar potential is also affected by light fermions. As for (ii), in non-minimal Higgs sectors it might be possible to compensate the effects of light fermions by changing the many parameters in the scalar sector accordingly without affecting any of the experimental constraints. However, this depends on the considered model and therefore should be justified case-by-case.

In this work we want to answer the question to which extent one of the simplest extensions of the SM which still features a strong first order EWPT and can be embedded into split SUSY. We consider a $2 \mathrm{HDM}$ with additional light $\mathrm{SU}(2)_{L} \otimes \mathrm{U}(1)_{Y}$ fermions and compute the strength of the EWPT taking into account temperature corrections due to the additional fermions. We compare the results with those obtained in the ordinary 2HDM and show that the fermion contributions can be large enough to turn an EWPT that would be weak in the pure 2HDM, into a strong first order EWPT. Furthermore, we demonstrate that in particular the parameter region of relatively heavy non-SM Higgs bosons can benefit from the additional fermions.

The paper is structured as follows. We introduce the model in section 2. The finite temperature corrections induced by the additional fermion degrees of freedom are discussed in section 3. Numerical results of the strength of the EWPT for the model are shown in section 4 and compared to results obtained in an ordinary 2HDM without additional fermions. A short outlook on possible split SUSY models is given section 5. We conclude in section 6 . 


\section{A 2HDM with electroweakinos}

In this paper, we work in the softly-broken $\mathbb{Z}_{2}$ symmetric version of the $2 \mathrm{HDM}[32,33]$

$$
\begin{aligned}
V_{2 \mathrm{HDM}}= & \frac{\lambda_{1}}{2}\left|\Phi_{1}\right|^{4}+\frac{\lambda_{2}}{2}\left|\Phi_{2}\right|^{4}+\lambda_{3}\left|\Phi_{1}\right|^{2}\left|\Phi_{2}\right|^{2}+\lambda_{4}\left|\Phi_{2}^{\dagger} \Phi_{1}\right|^{2} \\
& +\left(\frac{\lambda_{5}}{2}\left(\Phi_{1}^{\dagger} \Phi_{2}\right)^{2}-m_{12}^{2} \Phi_{1}^{\dagger} \Phi_{2}+\text { h.c. }\right)+m_{1}^{2}\left|\Phi_{1}\right|^{2}+m_{2}^{2}\left|\Phi_{2}\right|^{2},
\end{aligned}
$$

of type II, i.e. $\Phi_{1} / \Phi_{2}$ separately couple to the down/up-type sector of the SM fermions. All parameters in the Higgs potential in eq. (2.1) are real and hence there is no explicit CP-violation present in the Higgs potential. It is useful to define a basis with two Higgs doublets $H_{u}$ and $H_{d}$ with different isospin,

$$
H_{u}=\Phi_{2}, \quad H_{d}=-i \sigma_{2} \Phi_{1}^{*},
$$

such that $H_{u}, H_{d}$ and $\tan \beta=\left\langle H_{u}\right\rangle /\left\langle H_{d}\right\rangle$ coincide with the corresponding tree-level quantities of the MSSM. We have introduced here the mixing angle $\tan \beta$ as the ratio of the two vacuum expectation values of the neutral CP-even components of the Higgs doublets, denoted by the square brackets.

We extend the default particle content of the real CP-conserving $2 \mathrm{HDM}$ by additional non-SM like fermions. The BSM fermion sector should resemble the additional non-SM fermion part of the MSSM. Therefore, we will refer to the new fermions as electroweakinos in the following. The Lagrangian for the BSM fermions reads

$$
\begin{aligned}
-\mathcal{L}_{\text {fermion }}^{\mathrm{BSM}}= & \frac{1}{\sqrt{2}} H_{u}^{\dagger}\left(g_{2 u} \sigma_{a} \tilde{W}^{a}+g_{1 u} \tilde{B}\right) \tilde{H}_{u}-\frac{1}{\sqrt{2}} H_{d}^{\dagger}\left(g_{2 d} \sigma_{a} \tilde{W}^{a}+g_{1 d} \tilde{B}\right) \tilde{H}_{d} \\
& +\frac{M_{\tilde{W}}}{2} \tilde{W}^{a} \tilde{W}^{a}+\frac{M_{\tilde{B}}}{2} \tilde{B} \tilde{B}+\mu \tilde{H}_{u}\left(i \sigma_{2}\right) \tilde{H}_{d}+\text { h.c. },
\end{aligned}
$$

where the bino $\tilde{B}$ is gauged under $\mathrm{U}(1)_{Y}$ and the winos (higgsinos) $\tilde{W}\left(\tilde{H}_{u}, \tilde{H}_{d}\right)$ are triples (doublets) under $\mathrm{SU}(2)_{L}$. The Pauli-matrices are referred to as $\sigma_{a}(a=1,2,3)$. In contrast to the MSSM, the Yukawa couplings $g_{i j}$ with $i=1,2$ and $j=d$, $u$, and the Majorana masses $M_{\tilde{W}}, M_{\tilde{B}}, \mu$ are free input parameters. Note however, for $g_{1 u}=g_{1 d}=g_{1}^{\mathrm{MSSM}}$ and $g_{2 u}=g_{2 d}=g_{2}^{\mathrm{MSSM}}$, eq. (2.3) coincides with the corresponding part of the tree-level Lagrangian of the MSSM.

Diagonalizing eq. (2.3) yields four neutral mass eigenstates $\tilde{\chi}_{i}^{0}(i=1, \ldots, 4)$ with masses $m_{\tilde{\chi}_{1}^{0}} \leq \cdots \leq m_{\tilde{\chi}_{4}^{0}}$ and two charged mass eigenstates $\tilde{\chi}_{j}^{-}(j=1,2)$ with masses $m_{\tilde{\chi}_{1}^{-}} \leq m_{\tilde{\chi}_{2}^{-}}$,

$$
\begin{gathered}
\mathbf{m}_{\tilde{\chi}^{0}}=N^{*}\left(\begin{array}{cccc}
M_{\tilde{B}} & 0 & -\frac{v g_{1 d}}{2} c_{\beta} & \frac{v g_{1 u}}{2} s_{\beta} \\
& M_{\tilde{W}} & \frac{v g_{2 d}}{2} c_{\beta} & -\frac{v g_{2 u}}{2} s_{\beta} \\
& 0 & -\mu \\
\text { sym. } & 0
\end{array}\right) N^{\dagger}=\operatorname{diag}\left(m_{\tilde{\chi}_{1}^{0}}, \ldots, m_{\tilde{\chi}_{4}^{0}}\right) \\
\mathbf{m}_{\tilde{\chi}^{-}}=U^{*}\left(\begin{array}{cc}
M_{\tilde{W}} & \frac{v g_{2 d}}{\sqrt{2}} s_{\beta} \\
\frac{v g_{2 u}}{\sqrt{2}} c_{\beta} & \mu
\end{array}\right) V^{\dagger}=\operatorname{diag}\left(m_{\tilde{\chi}_{1}^{-}}, m_{\tilde{\chi}_{2}^{-}}\right),
\end{gathered}
$$


where $N, U$ and $V$ are unitary matrices and $c_{\beta} \equiv \cos \beta, s_{\beta} \equiv \sin \beta$. The abbreviation 'sym.' indicates that the matrix is symmetric with the corresponding entries to be filled in.

\section{$3 \quad$ Finite temperature corrections}

For the determination of the strength of the electroweak phase transition, we use the loop-corrected effective potential at finite temperature and follow the approach of $[9,34,35]$. The loop-corrected effective potential $V(T)$ at finite temperature splits into

$$
V(T)=V_{\text {tree }}+V_{\mathrm{CW}}+V_{\mathrm{T}}+V_{\mathrm{CT}},
$$

where $V_{\text {tree }}$ is the tree-level potential and given by eq. (2.1). The Coleman-Weinberg potential $V_{\mathrm{CW}}$ is the one-loop effective potential at zero temperature [36, 37] and $V_{\mathrm{T}}$ incorporates the temperature-dependent corrections [38, 39]. The counterterm potential $V_{\mathrm{CT}}$ is defined such that the next-to-leading order scalar (NLO) masses of the Higgs sector and the respective mixing angles are equal to the respective tree-level input parameters used in the parameter scan. This allows for an efficient parameter scan. A detailed discussion of the applied renormalization scheme is given in [34]. Furthermore, the derivation of the Coleman-Weinberg and of the temperature-dependent potential of the scalar sector is equivalent to the pure $2 \mathrm{HDM}$ and is presented in ref. [34]. Therefore, here we only discuss those parts of the calculation that change with respect to the pure 2HDM. The additional BSM fermions are taken into account by extending the summation of all degrees of freedom in the Coleman-Weinberg potential $V_{\mathrm{CW}}$ and the temperature dependent potential $V_{\mathrm{T}}$, respectively. The required zero-temperature masses $m_{\tilde{\chi}_{i}^{(0, \pm)}}$ are obtained by numerically diagonalizing the mass matrices in eq. (2.4). The dominant thermal corrections, the Daisy corrections, are treated in the Arnold-Espinosa method [40, 41]. These corrections are taken into account by replacing the temperature dependent potential with

$$
V_{\mathrm{T}} \rightarrow V_{\mathrm{T}}+V_{\text {Debye }},
$$

with the daisy or Debye corrections

$$
V_{\text {Debye }}=-\frac{T}{12 \pi}\left[\sum_{i=1}^{n_{\text {Higgs }}}\left(\left(\bar{m}_{i}^{2}\right)^{3 / 2}-\left(m_{i}^{2}\right)^{3 / 2}\right)+\sum_{a=1}^{n_{\text {gauge }}}\left(\left(\bar{m}_{a}^{2}\right)^{3 / 2}-\left(m_{a}^{2}\right)^{3 / 2}\right)\right],
$$

where the sum over the gauge bosons only extends over their longitudinal degrees of freedom. The tree-level masses $m_{i / a}^{2}$ are the eigenvalues obtained from the mass matrices $\Lambda_{(S)}^{i j}$ and $\Lambda_{(G)}^{a b}$ of the scalars $S$ and SM gauge bosons $G$, respectively. ${ }^{1}$ The thermal masses $\bar{m}_{i / a}^{2}$ are obtained by diagonalizing the mass matrices while including the respective thermal ring corrections $\Pi_{(S)}$ and $\Pi_{(G)}{ }^{2}$

$$
\begin{gathered}
\Lambda_{(S)}^{i j} \rightarrow \Lambda_{(S)}^{i j}+\Pi_{(S)}^{i j}, \\
\Lambda_{(G)}^{a b} \rightarrow \Lambda_{(G)}^{a b}+\Pi_{(G)}^{a b} .
\end{gathered}
$$

\footnotetext{
${ }^{1}$ We refer to ref. [42] for a detailed description of the notation of the mass matrices.

${ }^{2}$ The explicit formulae for $\Pi_{(S)}$ and $\Pi_{(G)}$ can be found in ref. [43].
} 
The additional fermion contributions to $\Pi_{S}$ and $\Pi_{G}$ are given by

$$
\begin{aligned}
\Pi_{\Phi_{i} \rightarrow \Phi_{j}}^{\text {inos }} & =\frac{T^{2}}{6}\left[\delta_{i 1} \delta_{j 1}\left(g_{1 d}^{2}+3 g_{2 d}^{2}\right)+\delta_{i 2} \delta_{j 2}\left(g_{1 u}^{2}+3 g_{2 u}^{2}\right)\right], \\
\Pi_{W_{i} \rightarrow W_{j}}^{\text {inos }} & =\delta_{i j} g_{2}^{2} \frac{T^{2}}{2}, \quad \Pi_{B \rightarrow B}^{\text {inos }}=g_{1}^{2} \frac{T^{2}}{6},
\end{aligned}
$$

where $g_{1}$ and $g_{2}$ are the $\mathrm{U}(1)_{Y}$ and $\mathrm{SU}(2)_{L}$ gauge couplings. The temperature-dependent corrections due to the fermions read

$$
V_{\mathrm{T}}^{\mathrm{inos}}=-\frac{T^{4}}{\pi^{2}} \operatorname{Tr}\left[J_{+}\left(\mathbf{m}_{\tilde{\chi}_{i}^{0}}^{2} / T^{2}\right)+2 J_{+}\left(\mathbf{m}_{\tilde{\chi}_{i}^{-}}^{2} / T^{2}\right)\right]+V_{\text {Debye }}^{\text {inos }}
$$

with the thermal integral

$$
J_{ \pm}(x)=\int_{0}^{\infty} d k k^{2} \log \left[1 \pm \exp \left(-\sqrt{k^{2}+x}\right)\right]
$$

where the '-' ('+') sign refers to bosons (fermions). In the decoupling limit of the electroweakinos

$$
M_{\tilde{B}}, M_{\tilde{W}}, \mu \rightarrow \infty \quad \text { and } \quad g_{i j} \rightarrow 0,
$$

one can deduce from eqs. (3.5) to (3.7) that all temperature corrections are heavily suppressed except for the ring corrections to the longitudinal degrees of freedom of the $\mathrm{SU}(2)_{L}$ and $\mathrm{U}(1)_{Y}$ gauge bosons. However, the derivation of the ring corrections [44] assumes the high-temperature limit $T \gg m_{i}$. In fact, the ring corrections vanish by construction in the decoupling regime. Therefore, the limit (3.8) has to be taken with care when performing a numerical study. Another critical limit is the one of large Yukawa couplings $g_{i j} \gg 1$. In this regime the Daisy resummation might also not be reliable anymore. Therefore, we expect the most reliable results for intermediate fermion masses and small and intermediate Yukawa couplings.

We utilize the computer code BSMPT $[43,45]$ for the numerical study which was extended to be able to calculate and minimize eq. (3.1) in the presence of additional non-SM like fermions. At the time of writing this publication, the extension of BSMPT is private but we plan to release this feature in an upcoming update of BSMPT.

\section{Phenomenology of the EWPT}

For the phenomenological analysis we start with the data sample collected in ref. [34], which studied the pure $2 \mathrm{HDM}$ i.e. eq. (2.1) without the inclusion of eq. (2.3). The sample has been reevaluated with ScannerS 2.0.0 [46, 47] which calculates Higgs decays using HDECAY [48-50] and applies the most recent collider constraints from HiggsBounds [51-55] and HiggsSignals [56-59] as well as theoretical constraints such as perturbative unitarity and stability of the scalar potential in the pure 2HDM. For a detailed description of ScannerS we refer to ref. [47]. The resulting dataset is valid for the pure 2HDM. In the next step, we investigate the impact of the additional non-SM-like fermions on the 
phenomenology of the EWPT. The strength of the EWPT is given by the ratio of the electroweak VEV $\omega_{c}$ at the critical temperature $T_{c}$,

$$
\xi_{c} \equiv \frac{\omega_{c}}{T_{c}}>1
$$

which is required to be larger than one for a sufficiently strong first order EWPT.

In general, the presence of additional light fermions also affects the Higgs boson branching ratios, electroweak precision data as well as other experimental constraints such as the dark matter relic density not considered in this work. For instance, the presence of light electroweakinos in an MSSM-inspired 2HDM and their influence on Higgs boson decays has been recently studied in ref. [60]. The authors find sizeable effects in some regions of the parameter space. However, for $\tan \beta \gtrsim 2$ and $m_{A} \gtrsim 650 \mathrm{GeV}$ they are out of the sensitivity of current experiments. This region with relatively large $m_{A}$, which disfavours a strong first order EWPT, is of particular interest for this work as discussed in section 4.1. Regarding electroweak precision data, it was shown in ref. [61] that light electroweakinos in the simplest split-SUSY scenario can even improve the electroweak precision fit. Likewise, theoretical issues such as the appearance of Landau poles at higher scales can be solved once the model considered in this work is connected to some more fundamental supersymmetric theory.

However, in this paper our focus is on the phenomenological impact of the additional light fermions on the EWPT rather than on collider physics and/or Dark Matter. A detailed study of the interplay between the different aspects is reserved for future work.

\subsection{The case $\xi_{c}^{2 \mathrm{HDM}}<1$}

We start the discussion with a sample point that does not feature a strong first order EWPT in the pure $2 \mathrm{HDM}$, i.e. it has $\xi_{c}^{2 \mathrm{HDM}}<1$ in the absence of $\mathcal{L}_{\text {fermion }}^{\mathrm{BSM}}$. The input parameters for the Higgs boson sector are

$$
\begin{aligned}
m_{h} & =125.09 \mathrm{GeV}, & m_{H} & =637.37 \mathrm{GeV}, \\
m_{A} & =811.35 \mathrm{GeV}, & m_{H^{ \pm}} & =839.90 \mathrm{GeV}, \\
\tan \beta & =6.15, & \alpha & =-0.1605
\end{aligned}
$$

The spectrum contains three rather heavy Higgs bosons above $600 \mathrm{GeV}$ and the heavier neutral CP-even Higgs boson $H$ mixes only by $\sim 2 \%$ with the SM-like Higgs boson $h$. Using these inputs we find for the strength of the EWPT

$$
\xi_{c}^{2 \mathrm{HDM}}=0.82
$$

when considering the pure $2 \mathrm{HDM}$ type II.

In the next step we assume $\mathcal{L}_{\text {fermion }}^{\mathrm{BSM}}$ to be present and calculate the strength of the EWPT for various scenarios of $g_{i j}$ and soft SUSY breaking mass parameters $M_{\tilde{W}} M_{\tilde{B}}$, and $\mu$. The first scenario considers fixed Yukawa couplings that are given by the $\mathrm{U}(1)_{Y}$ and $\mathrm{SU}(2)_{L} \mathrm{SM}$ gauge couplings, respectively, $g_{1 u}=g_{1 d}=g_{1}^{\mathrm{SM}}$ and $g_{2 u}=g_{2 d}=g_{2}^{\mathrm{SM}}$ while the soft SUSY breaking mass parameters are varied independently between $0.1-1 \mathrm{TeV}$. In figure 1 we plot the determined $\xi_{c}$ values as a function of the lightest charged and neutral 


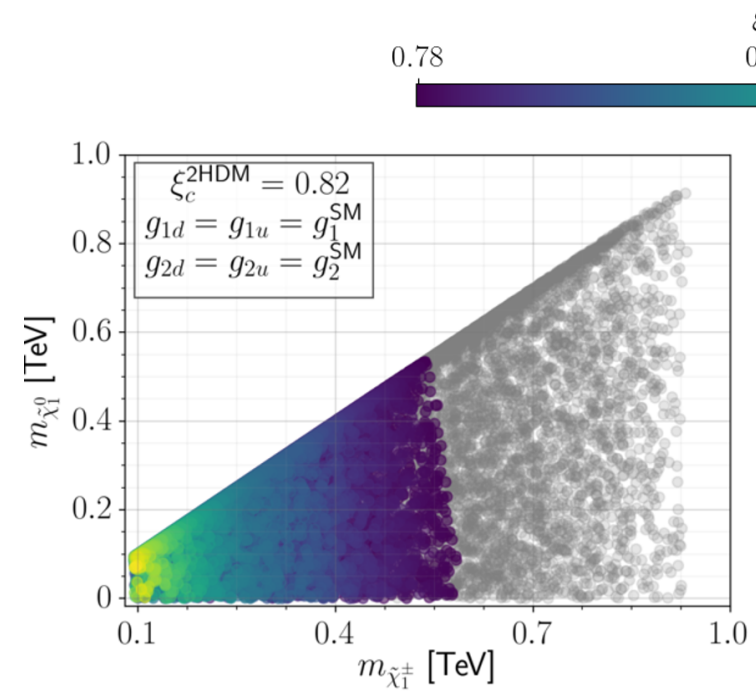

$\xi_{c}$

$0.94 \quad 1.09$

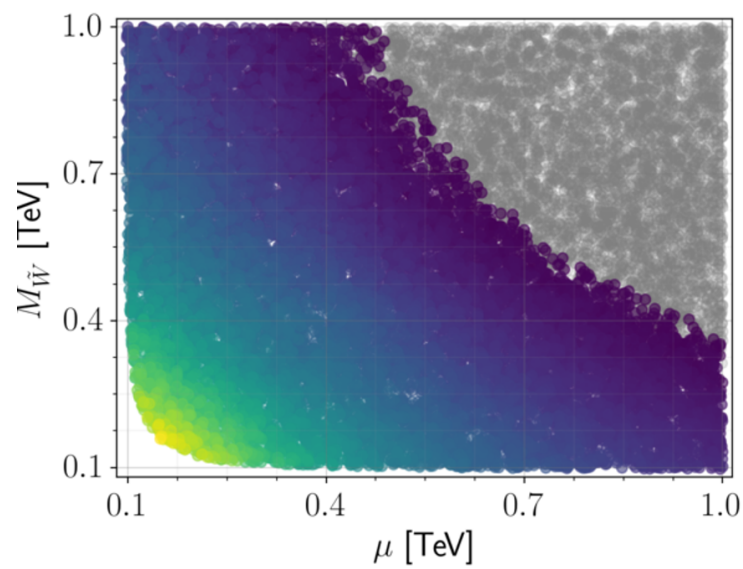

Figure 1. Prediction for $\xi_{c}$ for the parameter point defined in eq. (4.2) if only $M_{\tilde{B}}, M_{\tilde{W}}$ and $\mu$ are randomly varied. Left: as a function of the two lightest electroweakino masses. Right: as a function of $M_{\tilde{W}}$ and $\mu$.

electroweakino masses (left) and as a function of the input parameters $M_{\tilde{W}}$ and $\mu$ (right). The color code indicates the value of $\xi_{c}$ while grey points do not feature an NLO stable vacuum. We refer to a scenario to have an NLO stable vacuum if the global minimum of the one-loop effective potential at zero temperature coincides with the normal electroweak vacuum present at tree level. In this and all further plots we take the model-independent constraint $m_{\tilde{\chi}_{1}^{-}}>94 \mathrm{GeV}$ from LEP data into account [62] which effectively forbids the decay $h_{\mathrm{SM}} \rightarrow \tilde{\chi}_{1}^{+} \tilde{\chi}_{1}^{-}$. However, the region with $m_{\tilde{\chi}_{1}^{0}} \lesssim 60 \mathrm{GeV}$ in figure 1 (left) has to be taken with care as it would kinematically allow for the decay $h_{\mathrm{SM}} \rightarrow \tilde{\chi}_{1}^{0} \tilde{\chi}_{1}^{0}$. This additional constraint, however, does not affect the phenomenology of the EWPT since we also observe parameter points with $\xi_{c}>1$ and $m_{\tilde{\chi}_{1}^{0}}>\frac{m_{h_{\mathrm{SM}}}}{2}$ that feature a strong first order EWPT.

Figure 1 (left) indicates that additional light fermion degrees of freedom are able to induce a strong first order EWPT with $\xi_{c}>1$ for the considered parameter point which would otherwise have a smooth phase transition in a pure $2 \mathrm{HDM}$. Towards large fermion masses, however, $\xi_{c}$ becomes smaller and for masses $m_{\tilde{\chi}_{1}^{0, \pm}} \gtrsim 600 \mathrm{GeV}$ this benchmark scenario does not provide an NLO stable vacuum any more. We point out that this sharp cut in the mass plane is specific for this benchmark point and it is not observed in all parameter points.

In figure 1 (right) we see that also the $\mathrm{SU}(2)_{L}$-specific mass terms $M_{\tilde{W}}$ and $\mu$ show a strong correlation with $\xi_{c}$. On the other hand, $M_{\tilde{B}}$ (not shown here) does not show a strong correlation with $\xi_{c}$ in all investigated benchmark scenarios. Similar observations were made in ref. [63] which studied the EWPT within the SM extended by electroweakinos. The authors found that large Yukawa couplings of $g_{i j} \gtrsim \mathcal{O}(1.5)$ are required in order to achieve $\xi_{c}=1$, even in the limit of $\mu=M_{\tilde{W}}=0$. However, in the $2 \mathrm{HDM}$ considered in this work, $\mu \approx M_{\tilde{W}} \lesssim \mathcal{O}(150 \mathrm{GeV})$ is sufficient to induce a strong first order EWPT even if the electroweakinos are only weakly coupled to the Higgs bosons. 

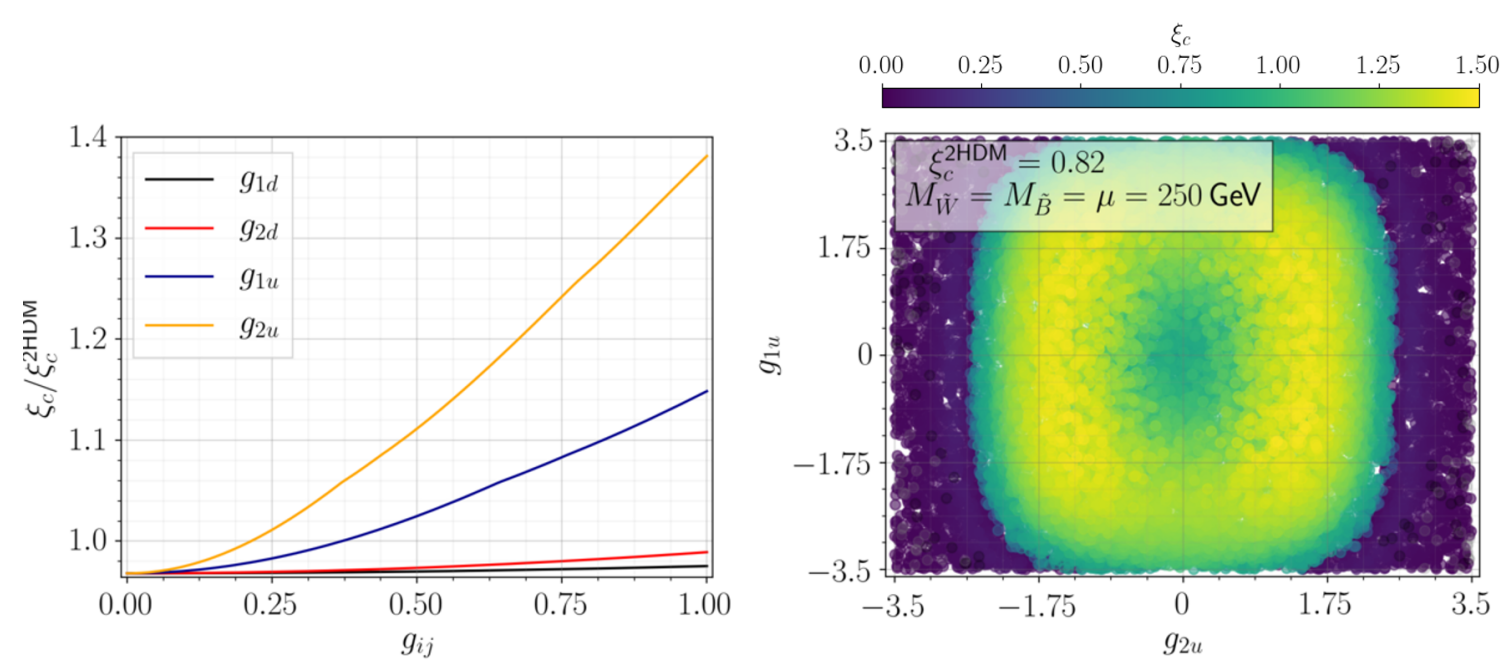

Figure 2. Prediction for $\xi_{c}$ for the parameter point defined in eq. (4.2) if only $g_{1 d}, g_{2 d}, g_{1 u}$ and $g_{2 u}$ are varied. Left: all four couplings are varied individually. Right: all couplings are varied simultaneously.

In the second scenario for $\xi_{c}^{2 \mathrm{HDM}}<1$, we consider fixed soft SUSY breaking mass parameters $M_{\tilde{W}}=M_{\tilde{B}}=\mu=250 \mathrm{GeV}$ and study the impact of the Yukawa couplings $g_{i j}$ on the EWPT. The variation of the Yukawa couplings alters the masses of the new light fermions and hence also the loop-corrected effective potential as well as the thermal integrals in eq. (3.6). Furthermore, a change of the Yukawa couplings also introduces a change in the additional temperature dependence through the Debye corrections given in eq. (3.5a). We investigate the dependence of $\xi_{c}$ normalized to $\xi_{c}^{2 \mathrm{HDM}}=0.82$ on the Yukawa couplings in figure 2 when varying them individually (left) from 0 to 1 and when varying them simultaneously (right) between -3.5 and 3.5. The left plot in figure 2 indicates that the Yukawa couplings $g_{2 i}(i=u, d)$ of the $\mathrm{SU}(2)_{L}$ fermions (yellow and red curves) have a stronger impact on the strength of the phase transition $\xi_{c}$ than their corresponding $\mathrm{U}(1)_{Y}$ couplings $g_{1 i}$ (blue and dark green curves). Overall the couplings to the up-type (i.e. SM-like) Higgs boson have a larger impact than those of the down-type Higgs boson (i.e. $\Phi_{1}$ ) with the largest impact of about $40 \%$ for $g_{2 u}=1$. The couplings to the down-type Higgs boson have only a negligible effect. The ratio $\xi_{c} / \xi_{c}^{2 \mathrm{HDM}}$ does not tend to 1 in the limit $g_{i j} \rightarrow 0$ because there are still non-vanishing contributions to the longitudinal vector boson, see eq. (3.5b).

In the right plot of figure 2 we vary all four Yukawa couplings simultaneously. Similar to the $\mathrm{U}(1)_{Y}$ mass parameter $M_{\tilde{B}}$, also the down-type couplings $g_{1 d}, g_{2 d}$ (not shown here) have only a minor impact on $\xi_{c}$. In contrast to the down-type couplings, the up-type couplings can shift $\xi_{c}$ from $\xi_{c}=0.8$ at $g_{i u}=0$ up to $\xi_{c}=1.4$ for $g_{i u}<1$. However, allowing for even larger Yukawa couplings $g_{i j}>1$ could potentially spoil perturbativity as well as significantly contribute to Higgs boson production rates via vector boson fusion and should be taken with care.

The interesting feature of the left plot in figure 2, that the $\mathrm{SU}(2)_{L}$ couplings have a stronger impact than the corresponding $\mathrm{U}(1)_{Y}$ couplings, is also seen in the right plot of 

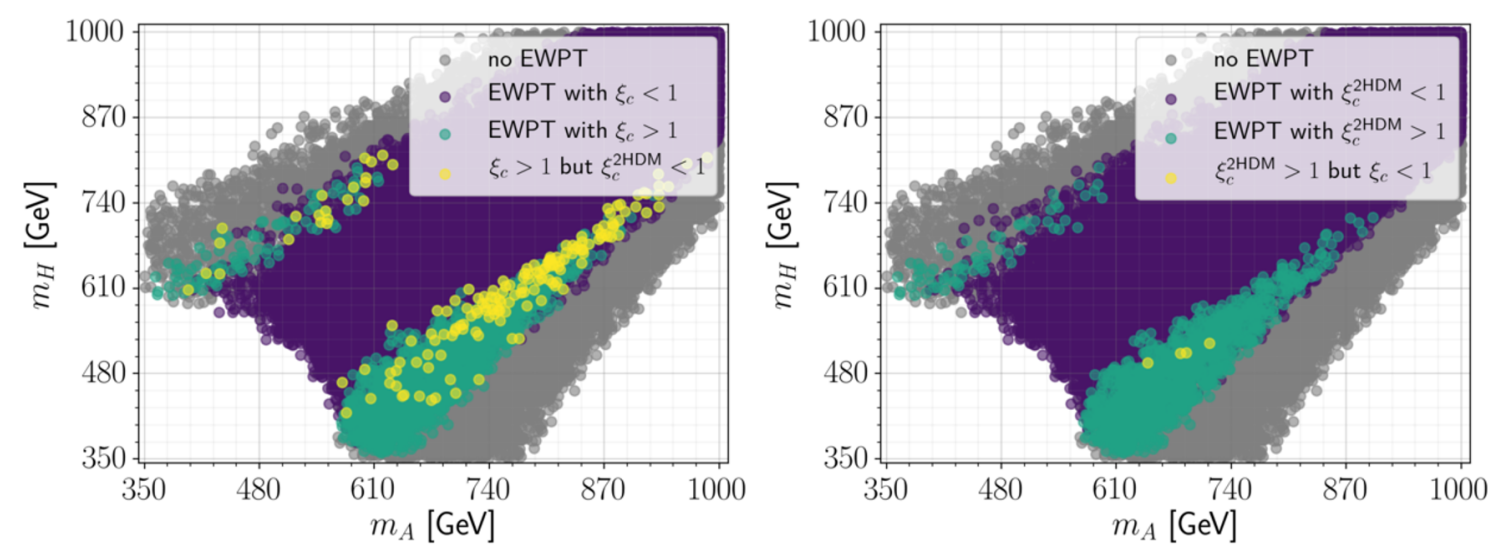

Figure 3. All points from the updated data sample of ref. [34] in the $\left(m_{H}, m_{A}\right)$-plane. Left: evaluated with the extended fermion sector. Right: evaluated in the pure 2HDM. Grey points do not yield an NLO stable electroweak vacuum, while violet (green) points have a phase transition with $\xi_{c}<1\left(\xi_{c}>1\right)$, respectively. Yellow points in the left figure feature a strong first order EWPT in the model with an extended fermion sector but not in the pure $2 \mathrm{HDM}$ and vice versa for the right plot.

figure 2. This behaviour can be explained with eq. (3.5), where the $\mathrm{SU}(2)_{L}$ contributions have larger prefactors since they contribute with more degrees of freedom. For very large Yukawa couplings above $g_{2 u}>2.5$, the strength of the EWPT $\xi_{c}$ is dropping rapidly to 0 .

The capability of the additional fermions to strengthen the EWPT compared to the $2 \mathrm{HDM}$ - or even pushing it into the strong regime - is not unique to the considered parameter point. In figure 3 (left), we show all points from the scan sample that are evaluated with the extended fermion sector fixed by $g_{1 u}=g_{1 d}=g_{1}^{\mathrm{SM}}, g_{2 u}=g_{2 d}=g_{2}^{\mathrm{SM}}$ and $M_{\tilde{B}}=M_{\tilde{W}}=\mu=200 \mathrm{GeV}$. The left plot shows the results in the $\left(m_{H}, m_{A}\right)$-plane when taking the additional fermions into account. The grey points do not yield an NLO stable electroweak vacuum, while the violet points develop an electroweak vacuum with $\xi_{c}<1$. Green as well as yellow points feature a strong first order EWPT, i.e. have $\xi_{c}>1$. Yellow points are of particular interest since they do not have a strong first order EWPT when considering the $2 \mathrm{HDM}$ without extended fermion sector, i.e. $\xi_{c}>1$ but $\xi_{c}^{2 \mathrm{HDM}}<1$. The latter points favour regions with larger $m_{A}$ and $m_{H}$ values. In this regime, the temperaturedependent corrections stemming from the Higgs sector are too small to reach $\xi_{c}^{2 \mathrm{HDM}}>1$ but the corrections from the fermions are large enough to finally achieve $\xi_{c}>1$.

In summary, non-SM like fermion degrees of freedom can be a helpful tool to weaken the tension between increasing Higgs boson mass constraints from collider experiments and the requirement of additional light scalar degrees of freedom to achieve a strong first order EWPT.

\subsection{The case $\xi_{c}^{2 \mathrm{HDM}}>1$}

Another interesting case is if a strong first order EWPT, $\xi_{c}^{2 \mathrm{HDM}}>1$, is achieved in the 2HDM but not within the $2 \mathrm{HDM}$ with an extended fermion sector. In the previous section, in figures 1 and 2 we have shown that additional fermions tend to strengthen rather then weaken the EWPT. In figure 3 (right) we investigate if there can still be cases where $\xi_{c}<1$ 
with additional fermions but $\xi_{c}^{2 \mathrm{HDM}}>1$ in the pure $2 \mathrm{HDM}$ (yellow points). It shows the same sample as in figure 3 (left) but evaluated in the $2 \mathrm{HDM}$. We find only few points for intermediate $\left(m_{A}, m_{H}\right)$ masses where this is the case. In addition, these cases have $\max \left\{\left|\xi_{c}-\xi_{c}^{2 \mathrm{HDM}}\right|\right\} \approx 10^{-3}$. This shows that the overall tendency of weakening the EWPT through the BSM fermions is rather tiny and hence negligible. In contrast, the yellow points in the left plot have $\max \left\{\left|\xi_{c}-\xi_{c}^{2 \mathrm{HDM}}\right|\right\} \approx 0.4$.

\section{$5 \quad$ Prospects for split-SUSY}

As already mentioned, the Yukawa interactions, $\mathcal{L}_{\text {fermion }}^{\text {BSM }}$, discussed in section 2 coincide with the corresponding tree-level Lagrangian of the MSSM. In addition, the considered type-II 2HDM can also be matched to the 2HDM. However, in the MSSM we have $\lambda_{5}=0$ at tree level. At the one-loop level, a matching of the MSSM to the 2HDM including light electroweakinos generates additional operators

$$
\left.V_{2 \mathrm{HDM}}\right|_{\text {loop }}=\Phi_{1}^{\dagger} \Phi_{2}\left(\frac{\lambda_{5}}{2} \Phi_{1}^{\dagger} \Phi_{2}+\lambda_{6} \Phi_{1}^{\dagger} \Phi_{1}+\lambda_{7} \Phi_{2}^{\dagger} \Phi_{2}\right)+h . c . .
$$

Integrating out heavy stops from the MSSM yields $\lambda_{5,6,7} \propto(4 \pi)^{-2} A_{t}^{2} / M_{\mathrm{SUSY}}^{2}$, where $A_{t}$ is the trilinear soft SUSY-breaking top Yukawa coupling. In split-SUSY, the soft-breaking stop masses $M_{\mathrm{SUSY}}$ are above the TeV scale and are currently bounded from below to roughly $1-2 \mathrm{TeV}[10]$. On the other hand, $A_{t}$ is at the electroweak scale since it is protected by the same symmetry as the soft SUSY-breaking gaugino masses [14]. Therefore, the loop-induced couplings cannot be much larger than $\lambda_{5,6,7} \approx 10^{-3}$. However, we find in our $2 \mathrm{HDM}$ scan $\lambda_{5} \geq 0.1$ to be a necessary condition for a strong first order EWPT if all other quartic couplings smaller than one (i.e. of similar order as in the MSSM). Considering that all other SUSY degrees of freedom, except for the electroweakinos, are too heavy to take part in the EWPT, one can exclude the possibility of a strong first order EWPT, since the effect of light electroweakinos is too small to compensate such a large discrepancy. This is also in agreement with the findings of ref. [64] which excluded a strong first order EWPT in the MSSM for stop masses above $115 \mathrm{GeV}$.

However, in non-minimal SUSY models such as the $\mathbb{Z}_{3}$-breaking ${ }^{3}$ NMSSM it is possible to generate sizeable quartic couplings $\lambda_{5,6,7}$ already at tree level by integrating out additional heavy singlets. This would enable to achieve a strong first order EWPT in SUSY for large stop and singlet masses while maintaining an effective and economic 2HDM near the SM scale. Furthermore, if the additional singlet fields are light, the low-energy theory might coincide with a $\mathbb{Z}_{2}$ breaking version of the singlet extended $2 \mathrm{HDM}[28]$ or a singlet extended SM $[24,30]$. While it is well known that additional light scalar singlets enrich the EWPT phenomenology, the effect of additional light fermions that are necessarily introduced when these models are motivated by split SUSY, has so far not been studied in great detail. In this paper, we have shown that the effect of light fermions on the strength of the EWPT is

\footnotetext{
${ }^{3}$ In general one can also generate a non-zero tree-level $\lambda_{5}$ in the scale-invariant NMSSM. However, this necessarily leads to a suppressed effective $\mu$-term, $\mu \rightarrow 0$ for $m_{\text {singlet }} \rightarrow \infty$ and is therefore not compatible with mass constraints on higgsinos.
} 
in general not negligible. However, it is still an open question whether the contributions from the fermions can be compensated by changes in other regions of the relatively large parameter space of non-minimal SUSY models. Therefore, a natural next step is to study the EWPT generated by non-minimal extended Higgs sectors in conjunction with extended fermion sectors, which we reserve for future works.

\section{Conclusions}

We studied the impact of additional light $\mathrm{SU}(2)_{L}$ and $\mathrm{U}(1)_{Y}$ fermions on the strength $\xi_{c}=v_{c} / T_{c}$ of the electroweak phase transition in the type-II $2 \mathrm{HDM}$ using an extended version of the computer code BSMPT. It was shown that $\xi_{c}$ can significantly differ from the value $\xi_{c}^{2 \mathrm{HDM}}$ of the corresponding $2 \mathrm{HDM}$ that does not involve additional fermions. Overall the strength of the EWPT changes in the presence of additional fermions by up to $40 \%$ compared to an ordinary 2HDM. The largest contribution is due to Yukawa couplings that couple $\mathrm{SU}(2)_{L}$ fermions to the up-type Higgs boson while bino-like states do have a large impact. Therefore, additional light fermions are a useful tool to weaken the tension between increasing mass constraints on BSM scalars and the requirement of additional light scalar degrees of freedom to accommodate a strong first order EWPT.

The findings can be of particular importance for non-minimal split SUSY models. While a strong first order EWPT in the (split) MSSM is already excluded, models beyond the MSSM can yield a 2HDM with additional light fermions - but enhanced quartic couplings - as a low-energy EFT. The impact of non-minimal extended Higgs and fermion sectors is an effect yet to be scrutinized.

\section{Acknowledgments}

M.G., M.M. and J.M. acknowledge support by the BMBF-Project $05 \mathrm{H} 18 \mathrm{VKCC} 1$, project number $05 \mathrm{H} 2018$.

Open Access. This article is distributed under the terms of the Creative Commons Attribution License (CC-BY 4.0), which permits any use, distribution and reproduction in any medium, provided the original author(s) and source are credited.

\section{References}

[1] ATLAS collaboration, Observation of a new particle in the search for the Standard Model Higgs boson with the ATLAS detector at the LHC, Phys. Lett. B 716 (2012) 1 [arXiv: 1207.7214] [INSPIRE].

[2] CMS collaboration, Observation of a New Boson at a Mass of $125 \mathrm{GeV}$ with the CMS Experiment at the LHC, Phys. Lett. B $\mathbf{7 1 6}$ (2012) 30 [arXiv:1207.7235] [InSPIRE].

[3] A.D. Sakharov, Violation of CP Invariance, $C$ asymmetry, and baryon asymmetry of the universe, Sov. Phys. Usp. 34 (1991) 392 [Pisma Zh. Eksp. Teor. Fiz. 5 (1967) 32] [InSPIRE].

[4] K. Kajantie, K. Rummukainen and M.E. Shaposhnikov, A Lattice Monte Carlo study of the hot electroweak phase transition, Nucl. Phys. B 407 (1993) 356 [hep-ph/9305345] [INSPIRE]. 
[5] Z. Fodor, J. Hein, K. Jansen, A. Jaster and I. Montvay, Simulating the electroweak phase transition in the SU(2) Higgs model, Nucl. Phys. B 439 (1995) 147 [hep-lat/9409017] [INSPIRE].

[6] K. Jansen, Status of the finite temperature electroweak phase transition on the lattice, Nucl. Phys. B Proc. Suppl. 47 (1996) 196 [hep-lat/9509018] [InSPIRE].

[7] K. Kajantie, M. Laine, K. Rummukainen and M.E. Shaposhnikov, The Electroweak phase transition: A Nonperturbative analysis, Nucl. Phys. B 466 (1996) 189 [hep-lat/9510020] [INSPIRE].

[8] WMAP collaboration, Nine-Year Wilkinson Microwave Anisotropy Probe (WMAP)

Observations: Final Maps and Results, Astrophys. J. Suppl. 208 (2013) 20 [arXiv:1212.5225] [INSPIRE].

[9] P. Basler, M. Mühlleitner and J. Müller, Electroweak Phase Transition in Non-Minimal Higgs Sectors, JHEP 05 (2020) 016 [arXiv: 1912.10477] [INSPIRE].

[10] CMS collaboration, Search for natural and split supersymmetry in proton-proton collisions at $\sqrt{s}=13 \mathrm{TeV}$ in final states with jets and missing transverse momentum, JHEP 05 (2018) 025 [arXiv: 1802.02110] [INSPIRE].

[11] A. Canepa, Searches for Supersymmetry at the Large Hadron Collider, Rev. Phys. 4 (2019) 100033.

[12] ATLAS collaboration, Search for electroweak production of supersymmetric states in scenarios with compressed mass spectra at $\sqrt{s}=13 \mathrm{TeV}$ with the ATLAS detector, Phys. Rev. D $\mathbf{9 7}$ (2018) 052010 [arXiv: 1712.08119] [inSPIRE].

[13] J.D. Wells, Implications of supersymmetry breaking with a little hierarchy between gauginos and scalars, in 11th International Conference on Supersymmetry and the Unification of Fundamental Interactions, (2003) [hep-ph/0306127] [INSPIRE].

[14] G.F. Giudice and A. Romanino, Split supersymmetry, Nucl. Phys. B 699 (2004) 65 [Erratum ibid. 706 (2005) 487] [hep-ph/0406088] [INSPIRE].

[15] N. Arkani-Hamed, S. Dimopoulos, G.F. Giudice and A. Romanino, Aspects of split supersymmetry, Nucl. Phys. B 709 (2005) 3 [hep-ph/0409232] [INSPIRE].

[16] A. Arvanitaki, C. Davis, P.W. Graham, A. Pierce and J.G. Wacker, Limits on split supersymmetry from gluino cosmology, Phys. Rev. D 72 (2005) 075011 [hep-ph/0504210] [INSPIRE].

[17] G. Bhattacharyya and T.S. Ray, Naturally split supersymmetry, JHEP 05 (2012) 022 [arXiv:1201.1131] [INSPIRE].

[18] H.E. Haber and R. Hempfling, The Renormalization group improved Higgs sector of the minimal supersymmetric model, Phys. Rev. D 48 (1993) 4280 [hep-ph/9307201] [InSPIRE].

[19] E. Bagnaschi, G.F. Giudice, P. Slavich and A. Strumia, Higgs Mass and Unnatural Supersymmetry, JHEP 09 (2014) 092 [arXiv: 1407.4081] [INSPIRE].

[20] G. Lee and C.E.M. Wagner, Higgs bosons in heavy supersymmetry with an intermediate $m_{A}$, Phys. Rev. D 92 (2015) 075032 [arXiv:1508.00576] [INSPIRE].

[21] E. Bagnaschi, J. Pardo Vega and P. Slavich, Improved determination of the Higgs mass in the MSSM with heavy superpartners, Eur. Phys. J. C 77 (2017) 334 [arXiv:1703.08166] [INSPIRE]. 
[22] H. Bahl and W. Hollik, Precise prediction of the MSSM Higgs boson masses for low $M_{A}$, JHEP 07 (2018) 182 [arXiv: 1805.00867] [INSPIRE].

[23] J. Braathen, M.D. Goodsell and P. Slavich, Matching renormalisable couplings: simple schemes and a plot, Eur. Phys. J. C 79 (2019) 669 [arXiv:1810.09388] [InSPIRE].

[24] M. Gabelmann, M. Mühlleitner and F. Staub, Automatised matching between two scalar sectors at the one-loop level, Eur. Phys. J. C 79 (2019) 163 [arXiv:1810.12326] [INSPIRE].

[25] P.J. Fox, G.D. Kribs and A. Martin, Split Dirac Supersymmetry: An Ultraviolet Completion of Higgsino Dark Matter, Phys. Rev. D 90 (2014) 075006 [arXiv:1405.3692] [InSPIRE].

[26] M. Gabelmann, M.M. Mühlleitner and F. Staub, The Singlet Extended Standard Model in the Context of Split Supersymmetry, Phys. Rev. D 100 (2019) 075026 [arXiv:1907.04338] [INSPIRE].

[27] R.T. Co, B. Sheff and J.D. Wells, The Race to Find Split Higgsino Dark Matter, arXiv:2105.12142 [INSPIRE].

[28] P. Athron, C. Balázs, A. Fowlie, G. Pozzo, G. White and Y. Zhang, Strong first-order phase transitions in the NMSSM - a comprehensive survey, JHEP 11 (2019) 151 [arXiv: 1908.11847] [INSPIRE].

[29] S.V. Demidov, D.S. Gorbunov and D.V. Kirpichnikov, Gravitational waves from phase transition in split NMSSM, Phys. Lett. B $\mathbf{7 7 9}$ (2018) 191 [arXiv:1712.00087] [INSPIRE].

[30] S.V. Demidov and D.S. Gorbunov, Non-minimal Split Supersymmetry, JHEP 02 (2007) 055 [hep-ph/0612368] [INSPIRE].

[31] S.V. Demidov, D.S. Gorbunov and D.V. Kirpichnikov, Split NMSSM with electroweak baryogenesis, JHEP 11 (2016) 148 [Erratum ibid. 08 (2017) 080] [arXiv:1608.01985] [INSPIRE].

[32] T.D. Lee, A Theory of Spontaneous T Violation, Phys. Rev. D 8 (1973) 1226 [InSPIRE].

[33] G.C. Branco, P.M. Ferreira, L. Lavoura, M.N. Rebelo, M. Sher and J.P. Silva, Theory and phenomenology of two-Higgs-doublet models, Phys. Rept. 516 (2012) 1 [arXiv:1106.0034] [INSPIRE].

[34] P. Basler, M. Krause, M. Muhlleitner, J. Wittbrodt and A. Wlotzka, Strong First Order Electroweak Phase Transition in the CP-Conserving 2HDM Revisited, JHEP 02 (2017) 121 [arXiv: 1612.04086] [INSPIRE].

[35] P. Basler, M. Mühlleitner and J. Wittbrodt, The CP-Violating 2HDM in Light of a Strong First Order Electroweak Phase Transition and Implications for Higgs Pair Production, JHEP 03 (2018) 061 [arXiv: 1711.04097] [INSPIRE].

[36] E.J. Weinberg, Radiative corrections as the origin of spontaneous symmetry breaking, Ph.D. thesis, Harvard University (1973) [hep-th/0507214] [INSPIRE].

[37] S.R. Coleman and E.J. Weinberg, Radiative Corrections as the Origin of Spontaneous Symmetry Breaking, Phys. Rev. D 7 (1973) 1888 [INSPIRE].

[38] L. Dolan and R. Jackiw, Symmetry Behavior at Finite Temperature, Phys. Rev. D 9 (1974) 3320 [INSPIRE].

[39] M. Quirós, Finite temperature field theory and phase transitions, in ICTP Summer School in High-Energy Physics and Cosmology, pp. 187-259 (1999) [hep-ph/9901312] [INSPIRE]. 
[40] P.B. Arnold and O. Espinosa, The Effective potential and first order phase transitions: Beyond leading-order, Phys. Rev. D 47 (1993) 3546 [Erratum ibid. 50 (1994) 6662] [hep-ph/9212235] [INSPIRE].

[41] P.B. Arnold, Phase transition temperatures at next-to-leading order, Phys. Rev. D 46 (1992) 2628 [hep-ph/9204228] [INSPIRE].

[42] J.E. Camargo-Molina, A.P. Morais, R. Pasechnik, M.O.P. Sampaio and J. Wessén, All one-loop scalar vertices in the effective potential approach, JHEP 08 (2016) 073 [arXiv: 1606.07069] [INSPIRE].

[43] P. Basler and M. Mühlleitner, BSMPT (Beyond the Standard Model Phase Transitions): A tool for the electroweak phase transition in extended Higgs sectors, Comput. Phys. Commun. 237 (2019) 62 [arXiv: 1803.02846] [INSPIRE].

[44] M.E. Carrington, The Effective potential at finite temperature in the Standard Model, Phys. Rev. D 45 (1992) 2933 [INSPIRE].

[45] P. Basler, M. Mühlleitner and J. Müller, BSMPT v2 a tool for the electroweak phase transition and the baryon asymmetry of the universe in extended Higgs Sectors, Comput. Phys. Commun. 269 (2021) 108124 [arXiv:2007.01725] [INSPIRE].

[46] R. Coimbra, M.O.P. Sampaio and R. Santos, ScannerS: Constraining the phase diagram of a complex scalar singlet at the LHC, Eur. Phys. J. C 73 (2013) 2428 [arXiv:1301.2599] [INSPIRE].

[47] M. Mühlleitner, M.O.P. Sampaio, R. Santos and J. Wittbrodt, ScannerS: Parameter Scans in Extended Scalar Sectors, arXiv:2007.02985 [INSPIRE].

[48] A. Djouadi, J. Kalinowski and M. Spira, HDECAY: A Program for Higgs boson decays in the standard model and its supersymmetric extension, Comput. Phys. Commun. 108 (1998) 56 [hep-ph/9704448] [INSPIRE].

[49] R. Harlander, M. Mühlleitner, J. Rathsman, M. Spira and O. Stål, Interim recommendations for the evaluation of Higgs production cross sections and branching ratios at the LHC in the Two-Higgs-Doublet Model, arXiv: 1312.5571 [INSPIRE].

[50] A. Djouadi, J. Kalinowski, M. Muehlleitner and M. Spira, HDECAY: Twenty++ years after, Comput. Phys. Commun. 238 (2019) 214 [arXiv:1801.09506] [INSPIRE].

[51] P. Bechtle, O. Brein, S. Heinemeyer, G. Weiglein and K.E. Williams, HiggsBounds: Confronting Arbitrary Higgs Sectors with Exclusion Bounds from LEP and the Tevatron, Comput. Phys. Commun. 181 (2010) 138 [arXiv:0811.4169] [InSPIRE].

[52] P. Bechtle, O. Brein, S. Heinemeyer, G. Weiglein and K.E. Williams, HiggsBounds 2.0.0: Confronting Neutral and Charged Higgs Sector Predictions with Exclusion Bounds from LEP and the Tevatron, Comput. Phys. Commun. 182 (2011) 2605 [arXiv:1102.1898] [InSPIRE].

[53] P. Bechtle, O. Brein, S. Heinemeyer, O. Stal, T. Stefaniak, G. Weiglein et al., Recent Developments in HiggsBounds and a Preview of HiggsSignals, PoS CHARGED2012 (2012) 024 [arXiv: 1301.2345] [INSPIRE].

[54] P. Bechtle, O. Brein, S. Heinemeyer, O. Stål, T. Stefaniak, G. Weiglein et al., HiggsBounds-4: Improved Tests of Extended Higgs Sectors against Exclusion Bounds from LEP, the Tevatron and the LHC, Eur. Phys. J. C 74 (2014) 2693 [arXiv:1311.0055] [InSPIRE].

[55] P. Bechtle, D. Dercks, S. Heinemeyer, T. Klingl, T. Stefaniak, G. Weiglein et al., HiggsBounds-5: Testing Higgs Sectors in the LHC 13 TeV Era, Eur. Phys. J. C 80 (2020) 1211 [arXiv: 2006.06007] [INSPIRE]. 
[56] P. Bechtle, S. Heinemeyer, T. Klingl, T. Stefaniak, G. Weiglein and J. Wittbrodt, HiggsSignals-2: Probing new physics with precision Higgs measurements in the LHC $13 \mathrm{TeV}$ era, Eur. Phys. J. C 81 (2021) 145 [arXiv: 2012.09197] [InSPIRE].

[57] P. Bechtle, S. Heinemeyer, O. Stål, T. Stefaniak and G. Weiglein, Probing the Standard Model with Higgs signal rates from the Tevatron, the LHC and a future ILC, JHEP 11 (2014) 039 [arXiv: 1403.1582] [INSPIRE].

[58] O. Stål and T. Stefaniak, Constraining extended Higgs sectors with HiggsSignals, PoS EPS-HEP2013 (2013) 314 [arXiv: 1310.4039] [INSPIRE].

[59] P. Bechtle, S. Heinemeyer, O. Stål, T. Stefaniak and G. Weiglein, HiggsSignals: Confronting arbitrary Higgs sectors with measurements at the Tevatron and the LHC, Eur. Phys. J. C $\mathbf{7 4}$ (2014) 2711 [arXiv: 1305.1933] [INSPIRE].

[60] H. Bahl, S. Liebler and T. Stefaniak, MSSM Higgs benchmark scenarios for Run 2 and beyond: the low $\tan \beta$ region, Eur. Phys. J. C 79 (2019) 279 [arXiv:1901.05933] [InSPIRE].

[61] S.P. Martin, K. Tobe and J.D. Wells, Virtual effects of light gauginos and higgsinos: A Precision electroweak analysis of split supersymmetry, Phys. Rev. D 71 (2005) 073014 [hep-ph/0412424] [INSPIRE].

[62] DELPHI collaboration, Search for charginos, neutralinos and gravitinos at LEP, Eur. Phys. J. C 1 (1998) 1 [inSPIRE].

[63] M. Carena, A. Megevand, M. Quirós and C.E.M. Wagner, Electroweak baryogenesis and new TeV fermions, Nucl. Phys. B $\mathbf{7 1 6}$ (2005) 319 [hep-ph/0410352] [INSPIRE].

[64] M. Carena, G. Nardini, M. Quirós and C.E.M. Wagner, The Baryogenesis Window in the MSSM, Nucl. Phys. B 812 (2009) 243 [arXiv:0809.3760] [INSPIRE]. 